\title{
Evaluation of Energy Harvesting Devices for Industrial Applications
}

\author{
Leander B. Hörmann ${ }^{1}$, Thomas Hölzl' ${ }^{1}$, Christian Kastl1, Peter Priller ${ }^{2}$, Andreas Springer ${ }^{3}$ \\ ${ }^{1}$ Linz Center of Mechatronics GmbH, 4040 Linz, Altenberger Straße 69, Austria, \\ ${ }^{2}$ AVL List GmbH, 8020 Graz, Hans-List-Platz 1, Austria, \\ 3 Institute for Communications Engineering and RF-Systems, Johannes Kepler University Linz, \\ 4040 Linz, Altenberger Straße 69, Austria \\ Leander.Hoermann@Icm.at
}

\begin{abstract}
:
The use of energy harvesting devices (EHDs), such as solar cells and thermoelectric generators, offers a promising opportunity for powering self-sufficient wireless devices. This becomes more important due to the miniaturization of electronic components and the resulting reduction in power consumption of integrated circuits. As a result, wiring and maintenance costs can be reduced or possibly omitted at all. However, the available output power of EHDs is highly dependent on the mounting location as well as on environmental conditions and may vary greatly over time. Therefore, it is meaningful to evaluate the EHDs at the location of use over a certain period of time in order to characterize them in real world scenarios. In this paper, we present a mobile and wireless measurement system for the characterization of EHDs. The measurement system enables the acquisition of different EHDs' characteristics for specific applications under various environmental conditions. This simplifies the selection of a suitable EHD and the design process of an energy management system for a particular application. An evaluation of the implemented measurement system has been carried out by characterizing a specific solar cell at different illumination levels for an industrial application.
\end{abstract}

Key words: Energy harvesting devices, characterization, wireless measurement system, industrial application, power supply evaluation.

\section{Introduction}

In the course of industrial internet-of-things (IloT), more and more sensors are applied at machines and processes to supervise and optimize them further. However, their wiring is a cost intensive task and reduces flexibility. It could be omitted if self-sufficient wireless sensor nodes are used. The wireless sensor nodes connect with each other to form a so-called wireless sensor network (WSN). Each sensor node measures physical quantities of industrial plants or processes and transmits them wirelessly towards a gateway using a certain communication protocol [1], [2]. The power supply of the wireless sensor nodes can be implemented in two different ways[3]:

First, the energy can be applied during the installation of the wireless sensor node by any kind of battery. Thus, the available energy for operation is limited and the operational time depends mainly on the average power consumption of the wireless sensor node and the capacity of the battery (neglecting any leakage currents and dynamic discharge behavior of the battery). The advantage is, that it allows the estimation of the operational time without any knowledge of the location of installation. For application scenarios with a specified operational time or if the device needs periodic physical maintenance, this solution could be more cost effective than the following solution.

Second, the power needed for supply can be harvested from the environment using so called energy harvesting devices (EHDs). EHDs can convert power of the environment into electrical power, for example solar cells or thermoelectric generators. Therefore, the operational time of the devices can be increased significantly. However, the harvestable electrical power of the environment is highly variable [4] and [5]. Therefore, an energy storage is needed to provide a continuous supply of the wireless sensor node. The energy storage can be implemented using for example rechargeable batteries or supercapacitors. If the capacity is well dimensioned, a perpetual operation is achievable [6]. 
Wireless sensor nodes supplied by energy harvesting have the advantage of a possible maintenance-free operation. As already said, the available output power of EHDs is highly dependent on the mounting location as well as on environmental conditions and may vary greatly over time. Therefore, it is meaningful to evaluate the EHDs at the location of use over a certain period of time in order to characterize them in real world scenarios. Using this realworld characterization data, an evaluation of continuous power supply can be performed. This paper presents a mobile and wireless measurement system for the characterization of EHDs. The measurement system enables the acquisition of different EHDs' characteristics for specific applications under various environmental conditions. This simplifies the selection of a suitable EHD and the design process of an energy management system for a particular application. The rest of the paper is organized as follows: First, this paper summarizes the industrial application scenario, second, it introduces the measurement system, and third, it discusses the measurement accuracy and shows first measurement results. Finally, it concludes the paper and outlines directions for future work.

\section{Application Scenario}

Testing of prototypes is an essential part in development and optimization of automotive powertrain systems. Prototypes are assembled and instrumented as unit-under-test (UUT) in an appropriate laboratory environment, such as a powertrain test bed. This allows observing properties in a realistically simulated environment. Such measurements help to characterize the UUTs to support further development. Wireless sensor nodes offer several advantages: First, a faster and simpler instrumentation due to elimination of cabling. Second, avoidance of plug contact problems and confusion that may occur with conventional instrumentation. And third, improvement of signal quality, because the analog signal is digitized directly at the node and all measuring points are electrically isolated from each other and from the automation system.

Energy supply of the wireless sensor nodes should not cause interruptions to the usual workflow. Any maintenance needs of nodes should be avoided during a measurement campaign (typically a few hours to several weeks). The wireless sensor nodes are therefore typically powered by batteries or energy harvesting systems (EHSs) [3] and thus severely energy constrained. They have either a limited amount of energy (batteries) or are prone to a low available power (EHSs) [7]. To be able to eval- uate the energy supply guarantee, it is needed to know average power consumption of the wireless sensor nodes and the available power over time. The following chapters presents the proposed measurement system for characterization of EHDs and first evaluation results.

\section{Measurement System for the Characteriza- tion of Energy Harvesting Devices}

The proposed measurement system is flexible and can be composed of several mobile measurement nodes for the simultaneous characterization of several EHDs at different locations as introduced in [8]. The autonomous measurement nodes consist of the developed measurement hardware and perform the characterization task. The characterization is based on a cyclic acquisition of the current-voltage characteristics of the connected EHDs. The measurement interval and the number of measurement points to be recorded can be configured. The measuring nodes form a mesh network with the base station and additional routing nodes and transmit the characterization data wirelessly to the base station. The thread radio protocol is used for communication. This is an IPv6-based mesh network protocol that is designed for secure, reliable and power-saving wireless communication [9]. OpenThread is used as open source software stack implementing the thread communication protocol.

The measurement hardware is powered by a rechargeable battery to enable mobile use and is designed for a characterization period of up to one year. Fig. 1 shows the simplified block diagram of the measurement hardware with the individual function blocks. The EHD to be characterized and external sensors for the acquisition of relevant environmental parameters are connected to the hardware. The supply circuit is responsible for the power supply of the other circuit components and provides the required voltage levels. The measurement hardware can either be supplied by an internal energy storage for mobile use or an external power supply. The internal energy storage is composed of a lithium-ion battery and a supercapacitor. In addition, it is possible to use the connected EHD to recharge the battery between the cyclic characterization measurements. This may extend the operational time of the measurement hardware significantly depending on available power.

The System-on-Chip (SoC) nRF52840 from Nordic Semiconductor is used as microcontroller and radio transceiver. This controller takes over the control of all hardware components, the power management, and provides the required IEEE802.15.4 radio interface for communication using OpenThread. 


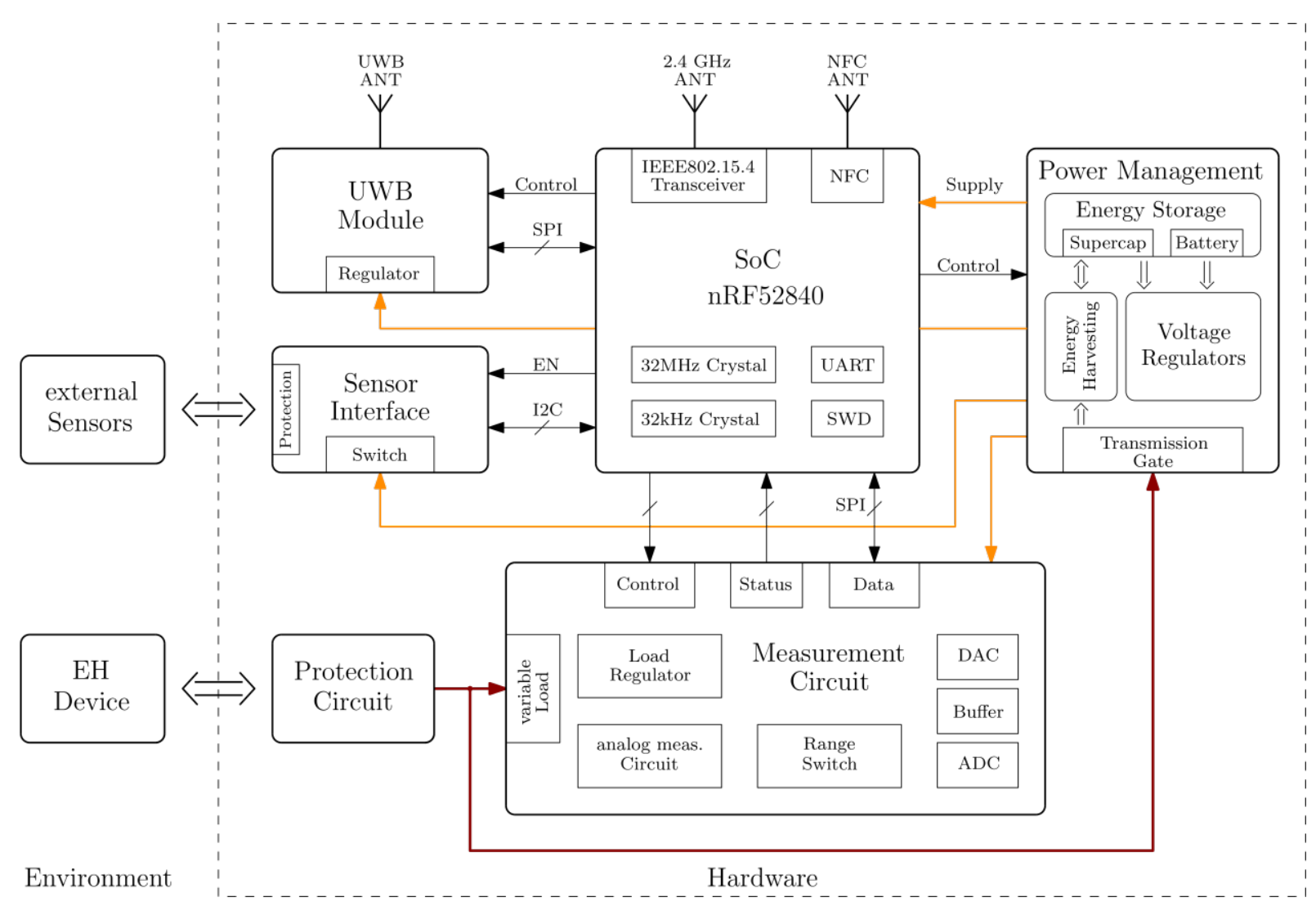

Fig.1: Block diagram of the proposed measurement hardware with connected external sensors and energy harvesting device.

The measurement circuit is responsible for recording the characteristics by measuring the current-voltage characteristic of the EHD. Using a variable load, different operating points including the short-circuit and open circuit case are set, and the resulting output currents and voltages of the source are measured. For a specific operating point, the output voltage of the EHD is controlled by the operating point controller to a desired preset value. The microcontroller uses a digital-to-analog converter (DAC) to provide the preset value. The measurements of the currents and voltages are carried out with an analog measurement circuit and an analog-to-digital converter (ADC). The measurable voltage ranges from below $1 \mathrm{mV}$ to up to $24 \mathrm{~V}$ and the measurable current ranges from below $1 \mu \mathrm{A}$ to up to $1 \mathrm{~A}$. In order to cover the wide measurement ranges with sufficient accuracy, the voltage measurement range is divided into two sub-ranges and the current measurement range into three sub-ranges. The change between the individual current measurement ranges is performed by an analog comparator circuit to ensure fast and automatic switching between the measurement ranges. The voltage measurement range is set by the microcontroller.
External sensors can be connected to the sensor interface for the acquisition of environmental parameters required for characterization, such as temperature or illumination level. A 2.5 $\checkmark$ supply voltage is provided for supplying the sensors and an $\mathrm{I}^{2} \mathrm{C}$ interface for communication. The sensor interface allows the simultaneous connection of several sensors, which enables the acquisition of different environmental parameters.

The ultra-wide-band (UWB) communication module is intended for a localization of the measurement hardware. This allows an automatic capturing of the position of the measurement node and a detection of any undesired or illegitimate movement. During normal operation, this hardware component is deactivated most of the time to keep the average power consumption as low as possible.

\section{Measurement Accuracy}

The evaluation of the measurement accuracy and calibration of the measurement hardware has been carried out using the reference instrument SourceMeter ${ }^{\circledR}$ 2602B from Keithley. Two calibration cycles have been performed with a temporal distance of four months. This enables an evaluation of the temporal drift of the measurement circuitry. During the calibra- 
Tab.1: Minimum and maximum average measurement deviation of the two voltage measurement ranges of the measurement hardware.

\begin{tabular}{|c|c|c|c|c|}
\hline \multirow{2}{*}{$\begin{array}{c}\text { Measurement } \\
\text { Range }\end{array}$} & \multicolumn{4}{|c|}{ Measurement deviation between measurement hardware and reference de- } \\
\cline { 2 - 5 } & $\Delta \mathrm{V}_{\max }(\mathrm{mV})$ & $\Delta \mathrm{V}_{\min }(\mathrm{mV})$ & $\Delta \mathrm{V}_{\max }(\%)$ & $\Delta \mathrm{V}_{\min }(\%)$ \\
\hline $1: 0-24 \mathrm{~V}$ & 0,98 & $-1,73$ & 0,004 & $-0,007$ \\
\hline $2: 0-4 \mathrm{~V}$ & 0,17 & $-0,60$ & 0,004 & $-0,015$ \\
\hline
\end{tabular}

Tab.2: Minimum and maximum average measurement deviation of the three current measurement ranges of the measurement hardware.

\begin{tabular}{|c|c|c|c|c|}
\hline \multirow{2}{*}{$\begin{array}{c}\text { Measurement } \\
\text { Range }\end{array}$} & \multicolumn{4}{|c|}{$\begin{array}{c}\text { Measurement deviation between measurement hardware and reference } \\
\text { instrument }\end{array}$} \\
\cline { 2 - 5 } & $\Delta I_{\max }$ & $\Delta I_{\min }$ & $\Delta I_{\max }(\%)$ & $\Delta I_{\min }(\%)$ \\
\hline $1: 10 \mathrm{~mA}-1 \mathrm{~A}$ & $0,33 \mathrm{~mA}$ & $-0,38 \mathrm{~mA}$ & 0,033 & $-0,038$ \\
\hline $2: 100 \mu \mathrm{A}-10 \mathrm{~mA}$ & $4,66 \mu \mathrm{A}$ & $-5,52 \mu \mathrm{A}$ & 0,047 & $-0,055$ \\
\hline $3: 0-100 \mu \mathrm{A}$ & $22 \mathrm{nA}$ & $-16 \mathrm{nA}$ & 0,022 & $-0,016$ \\
\hline
\end{tabular}

tion, different current and voltage values distributed over the individual measurement ranges were applied using the source of the reference instrument. The resulting currents and voltages are measured with the measurement hardware and the measurement unit of the reference instrument. Each measurement range is divided into 101 different measurement points, at which the current or voltage was applied. At each measurement point, 100 sample values are recorded with the measurement hardware and 250 values with the reference instrument. Using this data, a gain-offset calibration of each measurement range of the measurement hardware has been carried out. The mean values of the individual measurement points were used for the calibration and for the evaluation of the measurement deviations. The measurements were performed at room temperature. A statement about the noise performance of the measurement hardware is not possible with the used reference instrument since the noise is mainly determined by the noise behavior of the current or voltage sources of the reference instrument. In the following, the determined measurement accuracies of the measurement hardware are discussed for the supported voltage and current ranges.

Tab. 1 shows the absolute and relative deviations of the calibrated measurement hardware from the reference instrument for the two voltage measurement ranges. The relative deviations are referred to the respective upper range value. The manufacturer of the reference instrument specifies an accuracy of $\pm(0.015 \%+$ $1 \mathrm{mV}$ ) for the voltage measurement range up to $6 \mathrm{~V}$ and $\pm(0.015 \%+8 \mathrm{mV})$ for the measurement range up to $40 \mathrm{~V}$ [10]. The deviations of the calibrated circuit from the reference instrument are very small and lie within its measurement uncertainties.

The deviations of the three current measurement ranges of the calibrated measurement hardware are shown in Tab. 2. The relative measurement deviations are again related to the respective upper range value. The slightly increased deviations of measurement range 1 $(10 \mathrm{~mA}$ to $1 \mathrm{~A})$ and range $2(100 \mu \mathrm{A}$ to $10 \mathrm{~mA})$ are partly due to the slightly increased noise of the source of the reference instrument in these ranges. Despite this fact, a measurement error of well below $\pm 0.1 \%$ is achieved in all three measurement ranges. The manufacturer of the reference instrument specifies an accuracy of $\pm(0.02 \%+25 \mathrm{nA})$ for the current measurement range up to $100 \mu \mathrm{A}, \pm(0.02 \%+2.5 \mu \mathrm{A})$ for the measurement range up to $10 \mathrm{~mA}$ and $\pm(0.03 \%$ $+1.5 \mathrm{~mA}$ ) for the measurement range up to $1 \mathrm{~A}$ [10]. The minimum and maximum average deviations of the measurement hardware and 
Tab.3: Nominal performance parameters of the three evaluated solar cells for standard test conditions (STC).

\begin{tabular}{|c|c|c|c|c|c|c|c|}
\hline Solar cell type & $\begin{array}{c}\text { No. seri- } \\
\text { al cells }\end{array}$ & $\begin{array}{c}\text { Area } \\
\left(\mathrm{cm}^{2}\right)\end{array}$ & $\begin{array}{c}\mathrm{V}_{\mathrm{OC}} \\
(\mathrm{V})\end{array}$ & $\begin{array}{c}\text { Isc } \\
(\mathrm{mA})\end{array}$ & $\begin{array}{c}\mathrm{V}_{\mathrm{MPP}} \\
(\mathrm{V})\end{array}$ & $\begin{array}{c}\mathrm{I}_{\mathrm{MPP}} \\
(\mathrm{mA})\end{array}$ & $\begin{array}{c}\mathrm{P}_{\mathrm{MPP}} \\
(\mathrm{mW})\end{array}$ \\
\hline SLMD121H09L & 9 & 13.0 & 5.67 & 50.0 & 4.50 & 44.6 & 200.7 \\
\hline SM141K09L & 9 & 14.5 & 6.22 & 58.6 & 5.02 & 55.1 & 276.6 \\
\hline Solems07048032 & 7 & 15.4 & 5.00 & 12.0 & 3.90 & 9.9 & 38.6 \\
\hline
\end{tabular}

the reference instrument of the three current measurement ranges are very small and are in the order of magnitude of the measurement accuracy of the reference instrument.

\section{Evaluation Results of Energy Harvesting Devices}

Three different solar cells have been evaluated using the proposed measurement system, which are SLMD121H09L from IXYS Corporation [11], SM141K09L from IXYS Corporation [12], and Solems07048032 from SOLEMS S.A. [13]. Their nominal performance parameters for standard test conditions (STC) are shown in Tab. 3 . The STC are $25^{\circ} \mathrm{C}$ cell temperature, irradiance of $1000 \mathrm{~W} / \mathrm{m}^{2}$ and an air mass 1.5 (AM1.5) spectrum. This corresponds to direct sunlight on a clear day for a sun-facing $37^{\circ}$-tilted solar cell and a sun at an angle of $41.81^{\circ}$ above the horizon [14]. However, the application scenario targets an indoor application of the solar cells to supply wireless sensor nodes. Thus, the solar cells are evaluated under indoor equivalent illumination conditions which is typically less than $5 \%$ of STC (approx. 1000 to 2500 lux compared to 100.000 lux).

As reference light source we used an LED light source (Vibesta Capra 12B) with the spectrum shown in Fig. 2 (determined with Hamamatsu C11351-10 spectrometer).

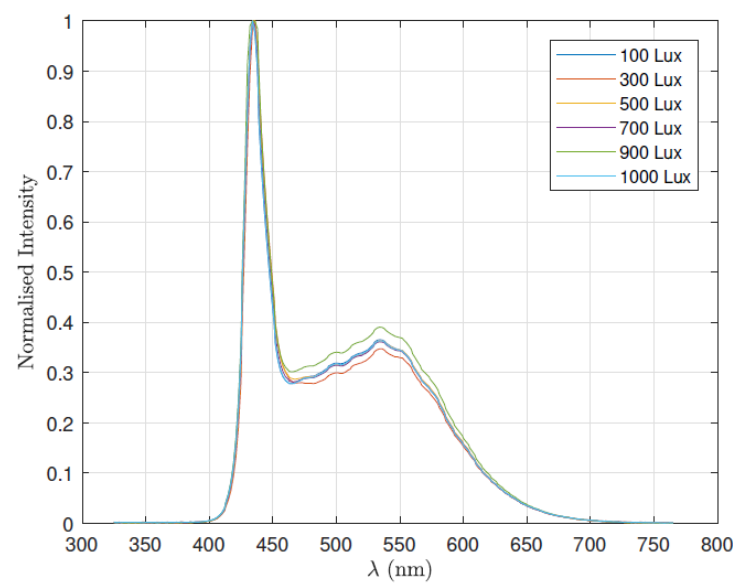

Fig. 2: Normalized spectrum of the reference light source at different illumination levels.
Fig. 3 and Fig. 4 shows the maximum power point (MPP) of the solar cells' power output densities at different illumination levels given in illuminance (Lux) and in irradiance $\left(\mathrm{mW} / \mathrm{cm}^{2}\right)$ respectively. The illuminance in Lux has been measured using the sensor OPT3001 from Texas Instruments [15]. The irradiance in $\mathrm{mW} / \mathrm{cm}^{2}$ has been measured using the sensor OPT3002 from Texas Instruments [16].

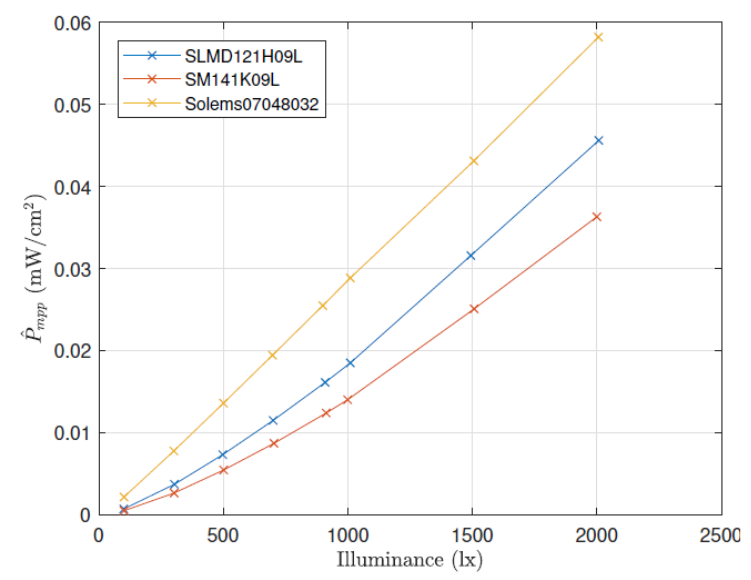

Fig. 3: Output power density of the three different solar cells depending on the illumination in Lux.

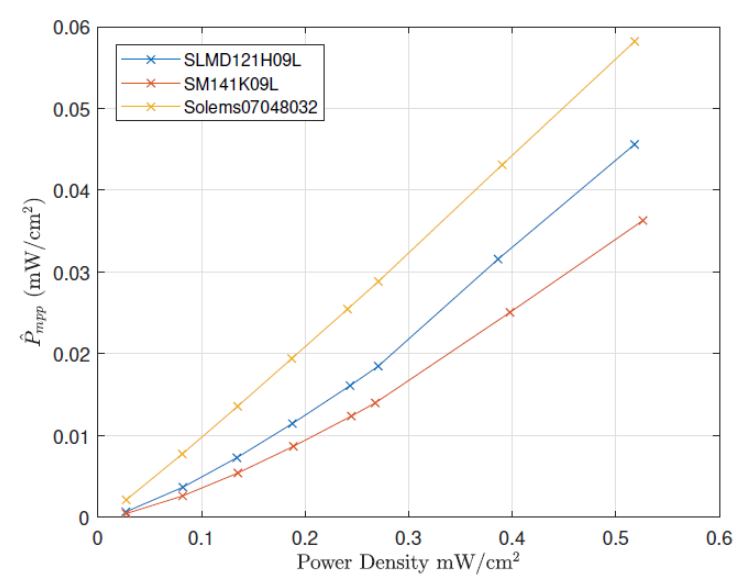

Fig. 4: Output power density of the three different solar cells depending on the irradiance power density in $\mathrm{mW} / \mathrm{cm}^{2}$.

Although the Solems07048032 has significant lower nominal performance parameter at STC, it performs best compared to the other two solar 
cells at low illumination levels. This is because it is developed especially for indoor applications.

Fig. 5 shows the open circuit voltage VOC over different illumination levels. It can be seen that the Solems07048032 has the highest output voltage, which results in its better performance regarding the MPP power output at low illumination levels.

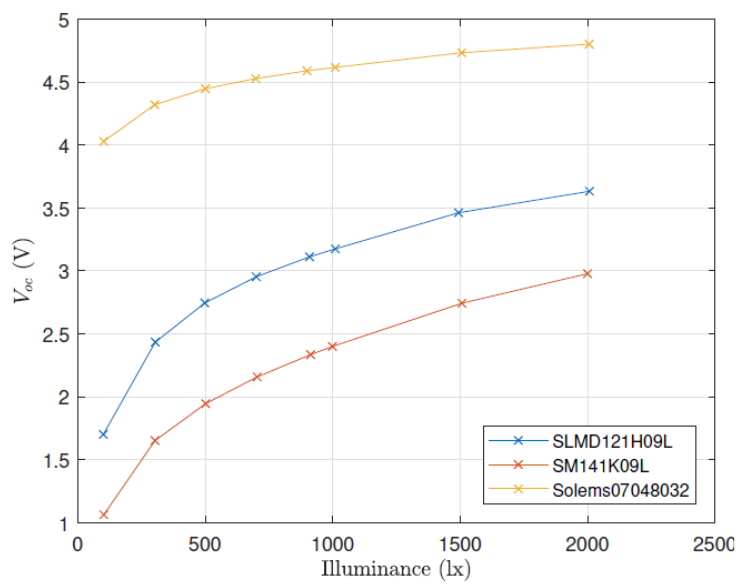

Fig. 5: Open-circuit voltage Voc of the three different solar cells over the illuminance in Lux.

Fig. 6 shows the ratio of the measured MPP voltage $V_{M P P}$ to the measured open circuit voltage $V_{o c}$ at different illumination levels.

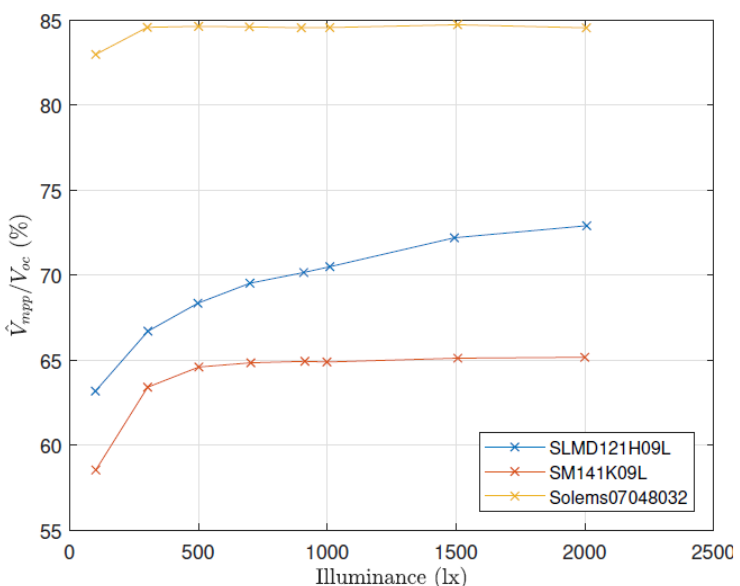

Fig. 6: Ratio of the measured MPP voltage $V_{M P P}$ to the measured open-circuit voltage $V_{O C}$ of the three different solar cells over the illuminance in Lux.

It can be seen that the measured ratios are significantly different to the ratios under STC (SLMD121H09L: 79.4\%, SM141K09L: 78\%, Solems07048032: $80.7 \%$ ). These different ratios have a direct influence on the design of the energy harvesting circuitry. Most power point trackers for embedded solutions on the market measure the open circuit voltage $V_{o c}$ and use a fixed ratio to set the MPP. Thus, they are no real MPP trackers, but it is a good and easy way to obtain an MPP approximation. However, this relies on the voltage ratio of the open circuit voltage $\mathrm{V}_{\mathrm{OC}}$ to the MPP voltage $\mathrm{V}_{\mathrm{MPP}}$. To optimize the MPP tracker's power output, the fixed ratio of $V_{M P P}$ to $V_{O C}$ must be set according to the measurements.

As shown above, the characterization data can be used first, to select the best solar cell for the given use case, and second, to adapt MPP trackers to the targeted solar cell and application scenario.

\section{Conclusion}

This paper presents a mobile and wireless measurement system for the characterization of EHDs. The measurement system enables the acquisition of different EHDs' characteristics for specific applications under various environmental conditions. This enhances the development process of energy harvesting wireless devices and enables a prediction of sufficient power supply. The evaluation of three solar cells reveals performance differences of the MPP power output and the ratio of MPP voltage to the open circuit voltage at low illumination levels. The results should be considered during design of energy harvesting systems in order to optimize the power supply.

Future work will target a long-term evaluation of different EHDs at different environmental conditions for industrial applications.

\section{Acknowledgement}

This work has been supported in part by the COMET-K2 Center of the Linz Center of Mechatronics (LCM) funded by the Austrian federal government and the federal state of Upper Austria.

\section{References}

[1] M. Bal, Industrial applications of collaborative wireless sensor networks: A survey, 2014 IEEE 23rd International Symposium on Industrial Electronics (ISIE), June 2014, pp. 1463-1468; doi: 10.1109/ISIE.2014.6864830

[2] H.-P. Bernhard, A. Springer, A. Berger, and P. Priller,"Life cycle of wireless sensor nodes in industrial environments," in 13th IEEE Int. Workshop Factory Commun. Sys., Trondheim, Norway, May 2017; doi: 10.1109/WFCS.2017.7991943

[3] L. B. Hörmann, T. Buchegger, and C. Steger, Optimizing the Energy Supply of Autonomous Wireless Sensor Nodes, Microelectronic Systems Symposium (MESS 2014), 2014, pp. 1-6; doi: 10.1109/MESS.2014.7010257

[4] A. Janek, C. Trummer, C. Steger, R. Weiss, J. Preishuber-Pfluegl, and M. Pistauer, Simulation based Verification of Energy Storage Architectures for Higher Class Tags Supported by Energy Harvesting Devices, 10th Euromicro Conference on Digital System Design Architectures, Methods 
and Tools (DSD 2007), Lubeck, 2007, pp. 463462.; doi: 10.1109/DSD.2007.4341510

[5] L.B. Hörmann, A. Berger, A. Pötsch, P. Priller, and A. Springer, "Estimation of the harvestable power on wireless sensor nodes," IEEE International Workshop on Measurements and Networking (MN 2015), 2015, 1-6; doi: $\underline{0.1109 / \text { IWMN.2015.7322964 }}$

[6] A. Kansal, D. Potter, and M. B. Srivastava, Performance Aware Tasking for Environmentally Powered Sensor Networks, Proceedings ofthe joint international conference on Measurement and modeling of computer systems, ser. SIGMETRICS '04/Performance '04, 2004, pp. 223-234; doi: 10.1145/1005686

[7] L. B. Hörmann, P. M. Glatz, K. B. Hein, M. Steinberger, C. Steger, and R. Weiss, "Towards an On-Site Characterization of Energy Harvesting Devices for Wireless Sensor Networks," in IEEE International Conference on Pervasive Computing and Communications Workshops (PERCOM Workshops 2012), 2012, pp. 415-418; doi: 10.1109/PerComW.2012.6197523

[8] T. Hölzl, "Drahtloses Messsystem für die Charakterisierung von Energy Harvesting Devices," master thesis, Institute for Communications Engineering and RF-Systems, Johannes Kepler University Linz, Austria, 2019.

[9] Thread Group, "Thread Overview (white paper)," revision 2.0, 2015, https://www.threadgroup.org/ Portals/0/documents/support/ThreadOverview 633 2.pdf , accessed April 2020.
[10] Tektronix, "2600B System SourceMeter ${ }^{\circledR}$ SMU Instruments," 2018, 112718.SBG 1KW-60906-0, https://www.tek.com/datasheet/smu-2650-serieshigh-power-sourcemeter/source-measure-unitssmu-instruments, accessed April 2020.

[11] IXYS Corporation, "IXYS SLMD121H09L - IXOLAR(TM) High Efficiency SolarMD Datasheet," Nov. 2016, http://ixapps.ixys.com/DataSheet/ SLMD121H09L Nov16.pdf, accessed April 2020.

[12] IXYS Corporation, "IXYS SM141K09L - IXOLAR(TM) High Efficiency SolarMD Datasheet," Sep. 2018, http://ixapps.ixys.com/DataSheet/ SM141K09L.pdf, accessed April 2020.

[13] SOLEMS S.A., "Thin-film solar cells for low and high illumination," Oct. 2017, https://www.solems. com/wp-content/uploads/Solar-cells en 17.pdf, accessed April 2020

[14] Sinovoltaics Group, "Standard Test Conditions (STC): definition and problems", 2011, https://sinovoltaics.com/learningcenter/quality/standard-test-conditions-stcdefinition-and-problems/, accessed April 2020.

[15] Texas Instruments, "OPT3001 Ambient Light Sensor (ALS)", SBOS681C, Nov. 2017, https://www.ti.com/lit/ds/symlink/opt3001.pdf, accessed April 2020.

[16] Texas Instruments, "'OPT3002 Light-to-Digital Sensor, SBOS745A, June2016, https://www.ti.com/lit/ds/symlink/opt3002.pdf, accessed April 2020. 